\title{
Liquid-Crystalline Phase Behavior of a Colloidal Rod-Plate Mixture
}

\author{
F. M. van der Kooij and H. N. W. Lekkerkerker \\ Van 't Hoff Laboratory for Physical and Colloid Chemistry, Debye Institute, Utrecht University, Padualaan 8, 3584 CH Utrecht, \\ The Netherlands \\ (Received 13 September 1999)
}

\begin{abstract}
The phase behavior of rod-plate mixtures was investigated using model systems containing unambiguously rod- and plate-shaped colloids. We find that the theoretically disputed biaxial nematic phase is unstable with respect to demixing into an isotropic and two uniaxial nematic phases. The phase behavior at very high densities is exceptionally rich and includes the coexistence of up to four different liquid crystalline phases, which stem from the coupling between the employed particle shapes and polydispersity.
\end{abstract}

PACS numbers: 82.70.Dd, 64.70.Md

The entropy-driven formation of the uniaxial nematic phase in suspensions of rodlike or platelike particles has been established in theory [1] and in simulations [2], as well as in experiments [3-6] in the course of this century. The probably even richer liquid crystalline phase behavior exhibited by mixtures of rodlike and platelike particles, on the other hand, is currently subject to debate. The central question here is whether or not there exists a so-called biaxial nematic in these mixtures, in which both rods and plates are orientationally ordered but in mutually perpendicular directions. In earlier theoretical studies this biaxial phase was found to be stable over a broad range of compositions [7-10]. Recent theoretical study [11] and simulations [12] show that, at least in some cases, the biaxial phase may be unstable with respect to demixing into two separate uniaxial phases of predominantly rods and plates, respectively. On the part of experimental evidence, indications for biaxiality have been reported by Yu and Saupe [13] for a system containing both cylindrical and lamellar micelles. There is, however, some doubt $[10,12]$ as to whether their observations are indicative of a biaxial phase transition or rather relate to a change in shape of the inherently "soft" micelles. In principle, model systems of inorganic colloids give the opportunity to study the phase behavior of unambiguously rodlike and platelike particles. In fact, the first experimental evidence for a uniaxial nematic phase was observed already in the 1920s in a suspension of inorganic vanadium pentoxide rods by Zocher [3]. However, a corresponding hard plate model system, i.e., consisting of particles with a short range repulsive interaction which seems crucial for exhibiting the unhindered isotropic to nematic $(I-N)$ phase transition $[14,15]$, has been lacking until recently. In this study we adopt a novel model system of platelike colloids which does show the $I-N$ transition in its unmixed form [6]. Combining this system with its rodlike analog allows us to investigate the liquid crystalline phase behavior of a mixture of such plainly rod- and plate-shaped particles.

The origin of liquid crystal stability in hard rod/plate mixtures is closely related to the case of the pure com- ponents, i.e., for rods and plates separately. Such spontaneous alignment in systems of nonattracting anisometric particles, which may seem counterintuitive at first thought, was first explained by Onsager [1] in the 1940s. He pointed out that the unarguable loss of orientational entropy associated with particle alignment in a nematic phase may, in fact, be more than compensated by a gain in packing entropy at higher particle concentrations. For mixtures of rods and plates, the Onsager theory has been extended by Stroobants and Lekkerkerker [9], yielding the following expression for the Helmholtz free energy of the particles:

$$
\begin{gathered}
\frac{\Delta F}{N k T}=\text { const }+\ln c+x_{r} \ln x_{r}+x_{p} \ln x_{p}+x_{r} \sigma_{r} \\
+x_{p} \sigma_{p}+c\left(x_{r}^{2} b_{r r} \rho_{r r}+2 x_{r} x_{p} b_{r p} \rho_{r p}\right. \\
\left.+x_{p}^{2} b_{p p} \rho_{p p}\right) .
\end{gathered}
$$

Here $N=N_{r}+N_{p}$ is the total number of particles, with $x_{r}=N_{r} / N$ the fraction of rods and $x_{p}=N_{p} / N$ the fraction of plates, and $c=N / V$ is the total number density. Furthermore, $\sigma_{r}$ and $\sigma_{p}$ reflect the orientational entropy of the rods and disks which are functionals of their orientational distribution function, while $\rho_{r r} b_{r r}, \rho_{r p} b_{r p}$, and $\rho_{p p} b_{p p}$ represent the orientational distribution function dependent excluded volumes of two rods, a rod and a plate, and two plates, respectively. From geometrical considerations it follows that these excluded volumes depend on the relative dimensions of both rods and plates. The subtle balance among the three entropy terms that appear in Eq. (1), related to mixing, orientation, and excluded volume, respectively, has raised a formidable challenge in predicting the phase behavior of rod/plate mixtures in theory and by simulations. Stroobants and Lekkerkerker studied the special case where all three excluded volumes are set equal. In addition to an isotropic phase and two uniaxial nematic phases of predominantly rods and plates, respectively, the phase diagram that they obtained does comprise a biaxial phase. A similar phase diagram was obtained in other theoretical studies $[7,8,10]$. The stability of the biaxial may, however, be less universal, as first suggested 
by van Roij and Mulder [11] who found demixing of the biaxial phase into two uniaxial nematic phases. The origin of the demixing lies in the generally larger excluded volume of a rod-plate pair as compared to the rod-rod and plate-plate excluded volume, which counteracts with the effect of mixing entropy that favors the biaxial phase. As the excess excluded volume gains importance at higher concentrations, resulting in the demixing, van Roij and Mulder showed that the phase diagram contains only a limited region of biaxial stability. This window of stability shrinks along with the aspect ratio of the particles, until it vanishes for aspect ratios smaller than about 9 . The role of orientational entropy is, however, partially neglected in their study as threefold discretized particle orientations were assumed. A limited region of biaxial stability was also found for mixtures of rods and plates with a common aspect ratio of 15 or 20 in a computer simulation [12].

Experimentally, the present study is based on the combination of two model systems, one consisting of rodlike boehmite $(\mathrm{AlOOH})$ colloids and the other of platelike gibbsite $\left(\mathrm{Al}(\mathrm{OH})_{3}\right)$ colloids. These systems were prepared by hydrothermal treatment (at $150{ }^{\circ} \mathrm{C}$ and $85^{\circ} \mathrm{C}$, respectively) of the same acidic aluminum alkoxide solution. Both systems were subsequently grafted with a modified polyisobutene ( $\mathrm{Mw} 1000 \mathrm{~g} / \mathrm{mole})$ steric stabilizer to provide the particles with approximately hard-core interactions when dispersed in apolar solvents like in this case toluene. A more detailed description of the synthesis of these suspensions can be found in Refs. $[16,17]$ for boehmite and Refs. $[6,18,19]$ for gibbsite. Characterization results for the particles are summarized in Table I. To study the phase diagram of mixtures of these systems, weighed amounts of the corresponding stock suspensions were homogenized in 2-mm cuvettes and left under thermostatic conditions in order to attain equilibrium. To establish the nature of the coexisting phases we used a combination of observation between crossed polarizers (for liquid crystalline textures), direct observation (for the degree of scattering and light absorption), and determination of the rod/plate number ratio displayed on transmission electron microscope (TEM) images of a particular phase.

TABLE I. Particle characterization results for the rods and plates used in this study. Particle sizes reflect the dimensions of the particle core as obtained from transmission electron microscopy (TEM). In the case of plates, the thickness was determined on deliberately flocculated samples to allow for edgewise observation by TEM. The given aspect ratios refer to the grafted particles, as determined from the ratio of the length over the thickness of the grafted particles while taking into account the approximate polymer thickness of $4 \mathrm{~nm}[20]$.

\begin{tabular}{lccc}
\hline \hline & Long axis $(\mathrm{nm})$ & Short axis $(\mathrm{nm})$ & Aspect ratio \\
\hline Rods & $160( \pm 30 \%)$ & $9( \pm 30 \%)$ & 10 \\
Plates & $200( \pm 25 \%)$ & $6( \pm 20 \%)$ & 15 \\
\hline \hline
\end{tabular}

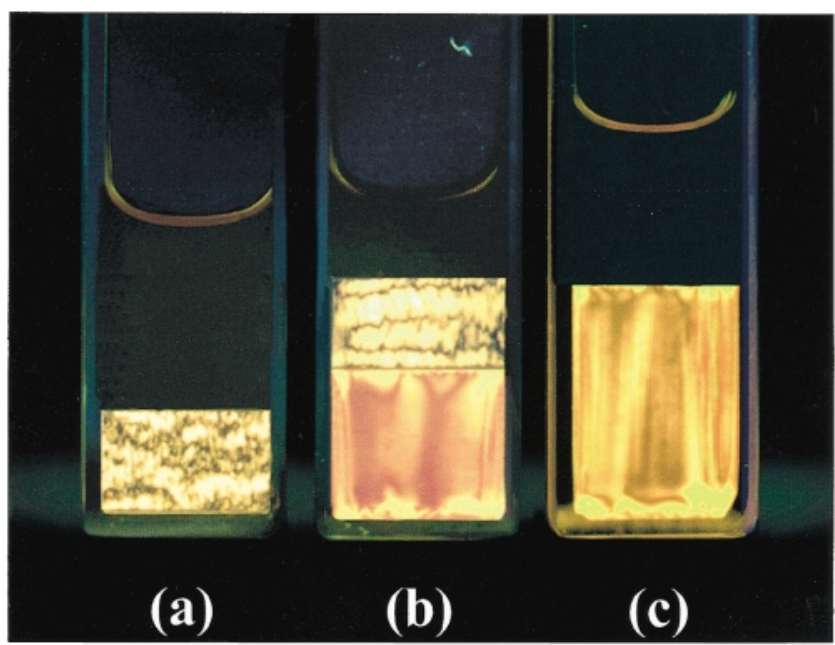

FIG. 1 (color). Phase separated rod/plate mixtures as observed between crossed polarizers. Depicted are (a) the $\left(I+N_{1}^{+}\right)$coexistence between an isotropic (top phase) and a rodlike nematic (bottom phase), (b) $\left(I+N_{1}^{+}+N_{1}^{-}\right)$coexistence between an isotropic (top phase), rodlike nematic (middle phase) and a platelike nematic (bottom phase), and (c) the $\left(I+N_{1}^{-}\right)$coexistence. Volume fractions of rods and plates, respectively, range from (a) $\{0.10,0.01\}$, (b) $\{0.10,0.08\}$, and (c) $\{0.02,0.18\}$.

Up to moderately high particle volume fractions $\left(\phi_{\text {total }} \leqq 0.3\right)$, phase separation of samples is macroscopically complete within 24 hours. Phase separation yields either an isotropic $(I)$ and a coexisting nematic phase $\left(N_{1}^{+}\right)$of predominantly rods, an isotropic and a coexisting nematic phase $\left(N_{1}^{-}\right)$of predominantly plates, or a $\left(I+N_{1}^{+}+N_{1}^{-}\right)$three-phase equilibrium, as depicted in Fig. 1. TEM micrographs of the three coexisting phases are shown in Fig. 2, which confirm the rodand plate-dominated nature of the $N_{1}^{+}$and $N_{1}^{-}$phases, respectively. These images also show that pronounced fractionation with respect to particle size has occurred. For instance, the average diameter of the plates in the $N_{1}^{-}$ phase is approximately $25 \%$ larger than in the coexisting $N_{1}^{+}$phase, while the polydispersity in diameter in the $N_{1}^{-}$ phase is reduced to less than $20 \%$. A prominent feature of the observed phase behavior is the complete absence of a biaxial phase. Unfortunately, it is not possible to draw a

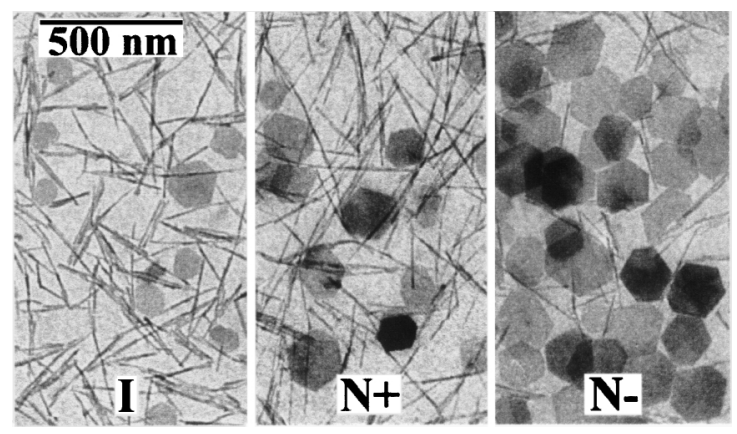

FIG. 2. TEM micrographs of samples taken from a $(I+$ $N_{1}^{+}+N_{1}^{-}$) phase separated rod/plate mixture. 


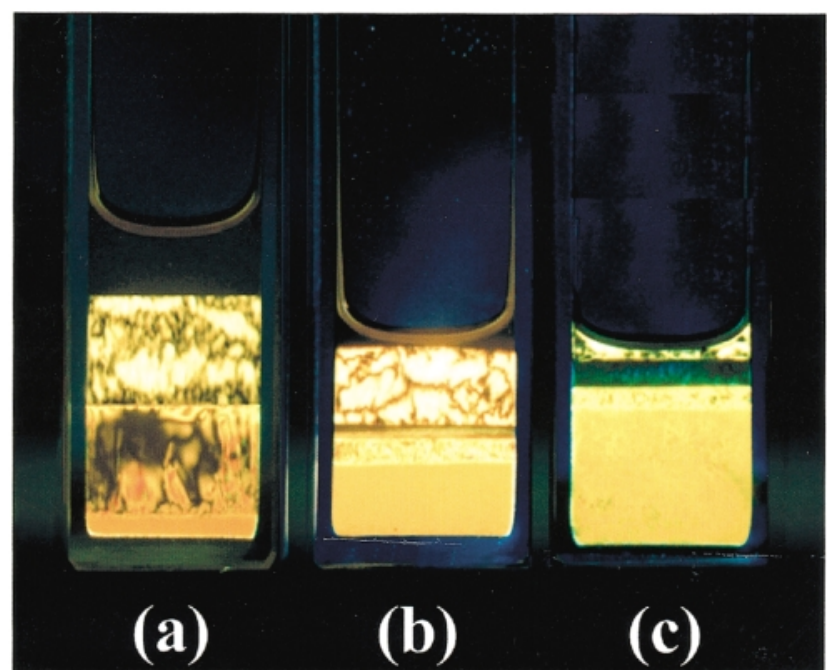

FIG. 3 (color). Phase separation in highly concentrated rod/ plate mixtures, as observed between crossed polarizers. Mentioned in the order of appearance from top to bottom, we identify the following coexistent phases: (a) $I+N_{1}^{+}+N_{1}^{-}+N_{2}^{-}$, (b) $I+N_{1}^{+}+N_{2}^{+}+N_{1}^{-}+N_{2}^{-}$, and (c) $N_{1}^{+}+N_{2}^{+}+N_{1}^{-}+$ $N_{2}^{-}$. Volume fractions of rods and plates, respectively, range from (a) $\{0.06,0.26\}$, (b) $\{0.10,0.22\}$, and (c) $\{0.07,0.29\}$.

comparison between our findings and the aforementioned micellar system showing signs of biaxiality [13], as the dimensions of the constituent particles of the latter are both unknown and dependent on the location in the phase diagram. However, the observed three-phase equilibrium adds experimental proof to the theoretically proposed demixing [11].

At very high concentrations $\left(0.3 \leqq \phi_{\text {total }} \leqq 0.5\right)$ the rod/plate phase behavior becomes notably rich (Figs. 3 and 4). While in principle one would expect the isotropic phase to vanish if the overall concentration of a $\left(I+N_{1}^{+}+N_{1}^{-}\right)$ sample is increased, we observe the appearance of two additional nematic phases before the isotropic phase eventually leaves the scene at very high concentration. With the observation of up to five coexisting phases, it follows

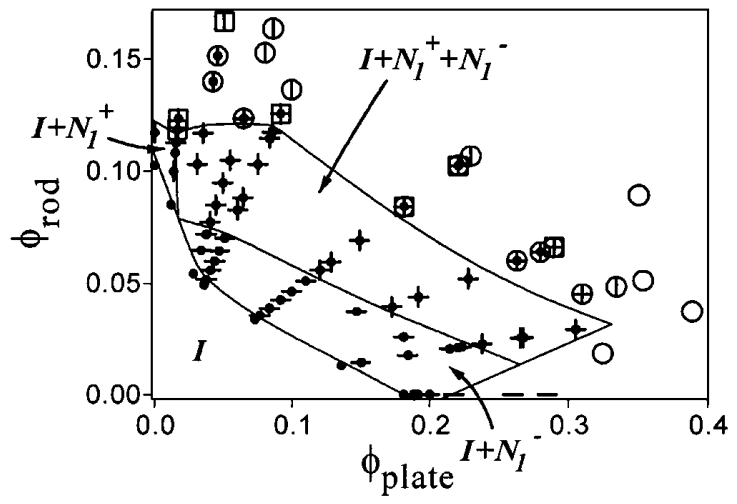

FIG. 4. The observed phase diagram for the rod/plate mixtures. Phases are coded as follows: $I(\mathbf{O}), N_{1}^{+}(\mid), N_{1}^{-}(-), N_{2}^{+}(\square)$, and $N_{2}^{-}(\bigcirc)$. Lines are drawn to be consistent with the phase equilibria they enclose. from the phase rule of Gibbs that in this concentration regime the polydisperse rod/plate mixtures studied can no longer be considered as a two-colloidal component system. Judged from the compositions of the four liquid crystalline phases, two of them being explicitly rod-rich and two explicitly plate-rich as indicated by electron micrographs of the different phases, we denominate the rod-rich nematics by $N_{1}^{+}, N_{2}^{+}$and the plate-rich nematics by $N_{1}^{-}$, $N_{2}^{-}$. Although phase separations take up to two weeks before being macroscopically complete, the positions of phase boundaries do reach a steady state. Furthermore, the boundaries are very sharp and phase volumina are both highly reproducible as well as coherently dependent on the point in the phase region. These observations suggest that phase separated samples have, indeed, reached a state of thermodynamical equilibrium.

In conclusion, we have studied the phase behavior of the first experimental system consisting of unambiguously rodlike and platelike particles with approximately hard core interactions. We find that, for the given particle dimensions, a biaxial phase is unstable with respect to demixing into two uniaxial nematic and one isotropic phase. At high particle concentrations the rod-plate mixtures show a richness of (liquid crystalline) phases which seems unequalled by any other colloidal system. One of the challenges in computer simulations would be to explore this high density regime, for instance, with respect to the stability of columnar and smectic phases in (monodisperse) mixed suspensions.

This work was supported by the Foundation for Fundamental Research on Matter (FOM) which is part of The Netherlands Organisation for the Advancement of Research (NWO).

[1] L. Onsager, Ann. N.Y. Acad. Sci. 51, 627 (1949).

[2] R. Eppenga and D. Frenkel, Mol. Phys. 52, 1303 (1984).

[3] H. Zocher, Anorg. Allg. Chem. 147, 91 (1925).

[4] J. D. Bernal and I. Fankuchen, J. Gen. Physiol. 25, 111 (1941).

[5] M.P. B. van Bruggen, F. M. van der Kooij, and H. N.W. Lekkerkerker, J. Phys. Condens. Matter 8, 9451 (1996).

[6] F. M. van der Kooij and H. N. W. Lekkerkerker, J. Phys. Chem. B 102, 7829 (1998).

[7] R. Alben, J. Chem. Phys. 59, 4299 (1973).

[8] Y. Rabin, W. E. McMullen, and W. M. Gelbart, Mol. Cryst. Liq. Cryst. 89, 67 (1982).

[9] A. Stroobants and H. N. W. Lekkerkerker, J. Phys. Chem. 88, 3669 (1984).

[10] A. Chrzanowska, Phys. Rev. A 58, 3229 (1998).

[11] R. van Roij and B. Mulder, J. Phys. (France) II 4, 1763 (1994).

[12] P. Camp, M.P. Allen, P.G. Bolhuis, and D. Frenkel, J. Chem. Phys. 106, 9270 (1997).

[13] L. J. Yu and A. Saupe, Phys. Rev. Lett. 45, 1000 (1980).

[14] A. Mourchid, A. Delville, and J. Lambard, Langmuir 11, 1942 (1995). 
[15] J. C. P. Gabriel, C. Sanchez, and P. Davidson, J. Phys. Chem. 100, 11139 (1996).

[16] P. A. Buining, C. Pathmamanoharan, J.B.H. Jansen, and H. N. W. Lekkerkerker, J. Am. Ceram. Soc. 74, 1303 (1991).

[17] P. A. Buining, Y. S. J. Veldhuizen, C. Pathmamanoharan, and H. N. W. Lekkerkerker, Colloids Surf. 64, 47 (1992).
[18] A. P. Philipse, A. M. Nechifor, and C. Patmamanoharan, Langmuir 10, 4451 (1994).

[19] A. Wierenga, T. A. J. Lenstra, and A. P. Philipse, Colloids Surf. A 134, 359 (1998).

[20] C. Smits, W. J. Briels, J. K. G. Dhont, and H. N.W. Lekkerkerker, Prog. Colloid Polym. Sci. 79, 287 (1989). 